\title{
Influência do periodo de quiescência dos ovos sobre o ciclo de vida de Aedes aegypti (Linnaeus, 1762) (Diptera, Culicidae) em condições de laboratório
}

\author{
Influence of eggs quiescence period on the Aedes aegypti (Linnaeus, 1762) \\ (Diptera, Culicidae) life cycle at laboratory conditions \\ Heloisa Helena Garcia da Silva e Ionizete Garcia da Silva
}

\begin{abstract}
Resumo Estudou-se a influência do período de quiescência dos ovos no ciclo de vida de Aedes aegypti (Linnaeus, 1762) (Diptera, Culicidae) em condições de laboratório, na busca de informações que possam melhorar o direcionamento das ações de controle, pois sabe-se que o ovo é a forma mais resistente do ciclo biológico, possibilitando ao mosquito ampla sobrevida, devido à resistência às adversidades climáticas. Os experimentos foram realizados numa câmara biológica, mantida à temperatura de $28 \pm 1^{\circ} \mathrm{C}$, com umidade relativa de $80 \pm 5 \%$ e fotofase de 12 horas. Apresentam-se os dados da influência de diferentes períodos de quiescência sobre a eclosão das larvas, desenvolvimento larval e pupal, ciclo evolutivo. Verificou-se o efeito altamente significativo do período de quiescência na eclosão das larvas. O período de quiescência não influenciou nas durações dos períodos de incubação, larval e pupal. Constatou-se que ovos de um mesmo período de quiescência apresentaram períodos de incubação estatisticamente diferentes entre si. As larvas eclodiam em grupos, definidos pela incubação, e este efeito de grupo foi significativo na duração do ciclo. Pode-se afirmar que, em 99,8\% dos ciclos, a variação foi determinada pela incubação.
\end{abstract}

Palavras-chaves: Quiescência. Aedes aegypti. Ciclo biológico. Dengue.

\begin{abstract}
The influence of the period of egg quiescence on the life cycle of Aedes aegypti (Linnaeus, 1762) (Diptera, Culicidae) was studied under laboratory conditions in order to improve the management of vector control. The eggs are known to be the most resistant stages during development, allowing a long survival of the mosquitoes under unfavorable climatic conditions. The experiments were performed in a biological chamber kept at $28 \pm 1^{\circ} \mathrm{C}$ temperature, with $80 \pm 5 \%$ relative humidity and 12 hours of photophase. Data about the influence of different periods of quiescence on eclosion, larval and pupal development and the developmentals cycle are presented. We observed a highly significant effect of the period of quiescence on larval eclosion. The period of quiescence had no influence on the duration of larval or pupal incubation. Eggs originating from the same period of quiescence showed significantly different periods of incubation The larvae emerged in groups defined by the period of incubation. This group effect was significant during the cycle. In $99.8 \%$ of the cycles the variation was determined by incubation.
\end{abstract}

Key-words: Quiescence. Aedes aegypti. Biological cycle. Dengue.

Instituto de Patologia Tropical e Saúde Pública (IPTSP) da Universidade Federal de Goiás (UFG). Goiânia, GO.

Apoio financeiro: FUNAPE proc.272, CNPq/RHAE proc.610100/95-5.

Endereço para correspondência: Profa. Heloisa Helena Garcia da Silva. Laboratório de Biologia e Fisiologia de Insetos, IPTSP/UFG,

Caixa Postal 131, 74001-970 Goiânia, GO. Fax: 5562 202-3066.

E-mail:ionizete@iptsp.ufg.br

Recebido para publicação em 21/5/98. 
Aedes aegypti distribui-se nas áreas tropical e subtropical do globo. Esse mosquito é o principal vetor dos quatro sorotipos do vírus do dengue que têm causado a dengue benigna e hemorrágica6 111213181930 31. Além disso, teve um papel importante como vetor da febre amarela urbana 510 , e no momento, devido a sua ampla distribuição geográfica no Brasil e América Latina, constitui-se numa ameaça à reurbanização da febre amarela.

Evidências faunística, primitiva e silvestre do A. aegypti na África indicam que esse mosquito surgiu naquele continente. É provável que esse mosquito tenha sido introduzido nas Américas pelas expedições colonizadoras ${ }^{10}$, posteriormente se dispersando com a movimentação humana. No Brasil, o $A$. aegypti foi erradicado e reintroduzido várias vezes. Atualmente esse mosquito distribui-se em 2.719 municípios brasileiros (dados da FNS/DF-1997), ocupando todas as regiões, com poucas perspectivas de ser controlado, trazendo consigo o dengue. $\mathrm{O}$ intrínseco relacionamento dos problemas de gerenciamento de programas, interrupções e limitação técnico-científica inviabilizam as ações de combate, deixando sem controle, tanto o mosquito quanto a doença, que até o momento, encontram-se em expansão8 1516172022252627 .

Por não dispormos de vacina efetiva até o momento, embora haja pesquisa14 1933 , progresso e perspectivas de obtenção, e nem de tratamento específico, o combate ao vetor é a medida mais eficiente na prevenção de surtos de dengue. Para isto, é imprescindível elaborar um programa de combate, sustentado nos conhecimentos de sua biologia, distribuição, comportamento, densidade. Nesse contexto, o fenômeno da quiescência dos ovos de A.aegytpi pode significar uma forma de recrudescimento e reinfestação do mosquito, em função da resistência às adversidades climáticas. Assim, espera-se que esses dados possam contribuir na reflexão, orientação ou direcionamento das ações de controle.

\section{MATERIAL E MÉTODOS}

O estudo da quiescência iniciou-se a partir de ovos de $A$. aegypti provenientes de uma ovoteca constituída de cartelas de ovos colhidas diariamente, durante um período de dois anos, e secadas por evaporação no ambiente da câmara. Dessa ovoteca retiraram-se 24 cartelas de ovos, que correspondiam a períodos de 3 a 720 dias de dessecação e armazenamento. Essas cartelas continham número variável de ovos, sendo aleatoriamente retiradas da ovoteca.

Os experimentos realizaram-se em uma câmara biológica climatizada 24 a $28 \pm 1^{\circ} \mathrm{C}$, com $80 \pm 5 \%$ de umidade relativa e fotofase cerca de $12 \mathrm{~h}$.

A incubação dos ovos de $A$. aegypti foi obtida a partir da imersão da cartela de ovos em água, examinada em intervalos de $2 \mathrm{~h}$ para verificar as eclosões das larvas. Essas observações foram feitas durante um período de 8 meses, mantendose o volume de água constante, com reposições diárias, e a limpeza das bacias feita em intervalos de 7 dias.

Após a eclosão, as larvas de 1ํestádio eram retiradas com o auxílio de uma pipeta de vidro, individualizadas em tubos de polietileno, transparentes, medindo $4,0 \mathrm{~cm}$ de altura $\times 4,7 \mathrm{~cm}$ de diâmetro, contendo $8 \mathrm{ml}$ de água. A reposição da água evaporada era feita, retirando-se a quantidade necessária de uma bacia plástica com água da rede pública de abastecimento, que ficava em repouso dentro da câmara. Com esse procedimento compatibilizava-se a temperatura da água com o ambiente climatizado, e, desta forma, evitava-se o choque térmico e, por conseguinte, a diapausa32. A alimentação era feita com ração para gatos 21 , triturada em gral e pistilo, e depois coada em tela fina, até se obterem finíssimas partículas. Com a ponta de uma espátula colocava-se, aproximadamente, $1,9 \mathrm{mg}$ de ração, em cada tubo. A reposição era feita quando se verificava diminuição ou desaparecimento da ração.

Para a alimentação das fêmeas usaram-se camundongos 24 , durante um período médio de $6 \mathrm{~h}$, em dias alternados. Os machos alimentavamse em algodão embebido de água açucarada21.

Para que as fêmeas realizassem a oviposição um copo com o interior revestido com papel filtro foi coberto com um cone de cartolina, cortado no ápice, criando um microambiente propício à postura, e impedindo também a morte de adultos, por afogamento.

Os adultos foram mantidos nas gaiolas de acasalamento e alimentados de acordo com técnica já definida21 24. Diariamente, procediase à limpeza das gaiolas, com papel toalha umedecido em água.

A análise de variância e o teste de Tukey, ao nível de 5\%, foram usados para comparação 
dos dados obtidos dos diversos períodos de quiescência. O teste de qui-quadrado de heterogeneidade foi usado na determinação da sexagem.

\section{RESULTADOS}

Das 24 amostras de ovos colocadas para incubar 11 apresentaram eclosão. Esta ocorreu em grupos com intervalos definidos pelo período de incubação.

Os resultados apresentados na Tabela 1 evidenciam o efeito altamente significativo do período de quiescência sobre o número de larvas eclodidas de $A$. aegypti, sendo que os períodos de 3 e 121 dias foram os mais favoráveis à eclosão.

Ao se calcular o coeficiente de determinação da incubação sobre o ciclo evolutivo, pode-se

Tabela 1 - Taxa de eclosão de larvas de Aedes aegypti, a partir de ovos com diferentes períodos de quiescência.

\begin{tabular}{|c|c|c|c|c|}
\hline $\begin{array}{l}\text { № do } \\
\text { grupo }\end{array}$ & $\begin{array}{l}\text { Quiescência } \\
\text { (dias) }\end{array}$ & $\begin{array}{c}\text { Total } \\
\text { de } \\
\text { ovos }\end{array}$ & $\begin{array}{c}\text { Média } \\
\text { eclosão } \\
\text { diária }\end{array}$ & $\begin{array}{c}\text { Eclosão } \\
(\%)\end{array}$ \\
\hline 1 & 3 & 807 & $86,1 \pm 51,12 a$ & 85,4 \\
\hline 2 & 32 & 698 & $5,3 \pm 0,98 b$ & 41,1 \\
\hline 3 & 63 & 586 & $6,4 \pm 2,13 b$ & 36,0 \\
\hline 4 & 91 & 738 & $12,1 \pm 4,76 \mathrm{c}$ & 47,7 \\
\hline 5 & 121 & 749 & $13,2 \pm 5,36 \mathrm{c}$ & 97,2 \\
\hline 6 & 154 & 800 & $1,6 \pm 0,30 d$ & 1,3 \\
\hline 7 & 273 & 612 & $8,6 \pm 3,78 \mathrm{bc}$ & 4,3 \\
\hline 8 & 337 & 611 & $1,0 \pm 0,00 d$ & 0,3 \\
\hline 9 & 427 & 842 & $5,6 \pm 0,25 b$ & 10,9 \\
\hline 10 & 462 & 800 & $1,0 \pm 0,00 d$ & 0,5 \\
\hline 11 & 492 & 1708 & $1,0 \pm 0,00 d$ & 0,2 \\
\hline
\end{tabular}

Obs: As médias seguidas da mesma letra, não apresentam diferença significativa entre si, pela análise de variância e o teste de Tukey, ao nível de $5 \%$.

afirmar que $99,8 \%$ da variação do ciclo foi determinada pela incubação (Tabela 2).

O período de quiescência não influenciou nas durações dos períodos larval e pupal (Tabela 3).
Verificou-se não haver efeito do período de quiescência na sexagem, mantendo-se sempre a proporção de emergência de 1 para 1, pelo teste do qui-quadrado de heterogeneidade, ao nível de $5 \%$. A existência de uma variável contínua

\begin{tabular}{|c|c|c|c|c|c|}
\hline \multirow{2}{*}{$\begin{array}{l}\text { № do } \\
\text { grupo }\end{array}$} & \multicolumn{5}{|c|}{ Quiescência (dias) } \\
\hline & 32 & 63 & 91 & 121 & 427 \\
\hline 1 & $2,0 \pm 0,31 a$ & $0,2 \pm 0,01 a$ & $0,3 \pm 0,01 a$ & $0,9 \pm 0,09 a$ & $0,7 \pm 0,11 a$ \\
\hline 2 & $6,6 \pm 0,36 b$ & $1,0 \pm 0,00 b$ & $0,8 \pm 0,05 b$ & $3,3 \pm 0,36 b$ & $2,0 \pm 0,01 b$ \\
\hline 3 & $19,0 \pm 0,68 c$ & $3,4 \pm 0,62 c$ & $3,6 \pm 1,30 c$ & $6,0 \pm 0,05 c$ & \\
\hline \multirow[t]{2}{*}{4} & $28,3 \pm 0,54 d$ & $14,4 \pm 1,41 d$ & $42,0 \pm 0,03 d$ & $32,5 \pm 0,24 d$ & \\
\hline & $32,3 \pm 0,90 \mathrm{e}$ & $43,9 \pm 0,10 \mathrm{e}$ & $81,0 \pm 0,02 e$ & $33,0 \pm 0,02 d$ & \\
\hline 6 & $38,7 \pm 0,62 f$ & $44,2 \pm 0,03 f$ & - & $33,0 \pm 0,00 \mathrm{e}$ & \\
\hline 7 & $85,2 \pm 0,02 g$ & - & - & $42,4 \pm 0,13 f$ & \\
\hline 8 & - & - & - & $53,2 \pm 0,09 g$ & \\
\hline 9 & - & - & - & $54,6 \pm 0,09 g$ & \\
\hline 10 & - & - & - & $63,0 \pm 0,06 \mathrm{~h}$ & \\
\hline 11 & - & - & - & $64,4 \pm 0,02 i$ & \\
\hline 12 & - & - & - & $74,0 \pm 0,44 j$ & \\
\hline 13 & - & - & - & $79,5 \pm 0,11 \mathrm{k}$ & \\
\hline
\end{tabular}

Obs: As médias seguidas da mesma letra, não apresentam diferença significativa entre si, pelo teste de Tukey, ao nível de $5 \%$. 
Tabela 3 - Duração do período larval e pupal (pós-embrinário) de Aedes aegypti, a partir de ovos com diferentes períodos de quiescência.

\begin{tabular}{|c|c|c|c|c|c|}
\hline \multirow{2}{*}{$\begin{array}{l}\text { No do } \\
\text { grupo }\end{array}$} & \multicolumn{5}{|c|}{ Quiescência (dias) } \\
\hline & 32 & 63 & 91 & 121 & 427 \\
\hline 1 & $9,5 \pm 0,33 a$ & $9,8 \pm 0,32 a$ & $9,6 \pm 0,31 a$ & $9,1 \pm 0,29 a$ & $10,3 \pm 0,39 a$ \\
\hline 2 & $8,7 \pm 0,33 a b$ & $8,7 \pm 0,27 b$ & $8,9 \pm 0,28 a b$ & $7,9 \pm 0,28 b$ & $8,8 \pm 0,42 b$ \\
\hline 3 & $8,0 \pm 0,27 b$ & $8,6 \pm 0,26 b c$ & $7,9 \pm 0,25 c$ & $8,0 \pm 0,34 b c$ & - \\
\hline 4 & $8,4 \pm 0,25 a b$ & $8,8 \pm 0,29 b$ & $7,8 \pm 0,30 c$ & $8,4 \pm 0,28 a b c$ & - \\
\hline 5 & $9,3 \pm 0,34 a b$ & $7,8 \pm 0,25 \mathrm{~cd}$ & $8,2 \pm 0,22 b c$ & $8,7 \pm 0,32 a b c$ & - \\
\hline 6 & $8,1 \pm 0,14 b$ & $7,7 \pm 0,25 d$ & - & $8,8 \pm 0,32 \mathrm{ac}$ & - \\
\hline 7 & $8,8 \pm 0,25 a b$ & - & - & $8,1 \pm 0,29 b c$ & - \\
\hline 8 & - & - & - & $8,5 \pm 0,29 b c$ & - \\
\hline 9 & - & - & - & $7,8 \pm 0,29 b c$ & - \\
\hline 10 & - & - & - & $9,3 \pm 0,26 \mathrm{ad}$ & - \\
\hline 11 & - & - & - & $7,9 \pm 0,22 b c$ & - \\
\hline 12 & - & - & - & $10,0 \pm 0,44 d$ & - \\
\hline 13 & - & - & - & $9,4 \pm 0,29 \mathrm{ad}$ & - \\
\hline
\end{tabular}

Obs: As médias seguidas da mesma letra, não apresentam diferença significativa entre si, pelo teste de Tukey, ao nível de 5\%.

- período de quiescência - justificou o uso do teste de tendência, para verificar se a proporção de machos e fêmeas aumentava ao longo dos períodos, mas esse efeito não foi detectado.

O período de quiescência não exerceu influência na longevidade de machos e fêmeas de $A$. aegypti (Tabelas 4 e 5). A longevidade de fêmeas e machos foi estatisticamente igual entre os primeiros ciclos de todos os períodos de quiescência. Constatou-se influência significativa do grupo na longevidade das fêmeas, no período de 121 dias de quiescência (Tabela 4). As fêmeas apresentaram longevidade significativamente maior do que a dos machos.

Tabela 4 - Longevidade média de fêmeas de Aedes aegypti, em relação a diferentes períodos de quiescência.

\begin{tabular}{|c|c|c|c|c|c|c|c|}
\hline \multirow{2}{*}{$\begin{array}{l}\text { № do } \\
\text { grupo }\end{array}$} & \multicolumn{7}{|c|}{ Quiescência (dias) } \\
\hline & 3 & 32 & 63 & 91 & 121 & 154 & 427 \\
\hline 1 & $47,6 \pm 3,5$ & $47,7 \pm 3,2 a$ & $39,4 \pm 2,8 a$ & $44,0 \pm 6,1$ & $50,9 \pm 3,2 a$ & $46,0 \pm 6,4$ & $43,8 \pm 2,9$ \\
\hline 2 & - & $46,9 \pm 2,38 a$ & $32,5 \pm 2,26 a$ & $49,0 \pm 2,31 a$ & $27,6 \pm 3,95 b$ & - & - \\
\hline 3 & - & $43,0 \pm 10,85 a$ & - & $51,5 \pm 7,93 a$ & $57,8 \pm 4,85 a$ & - & - \\
\hline 4 & - & $29,7 \pm 10,54 a$ & - & - & $55,9 \pm 6,88 a$ & - & - \\
\hline 5 & - & - & - & - & $49,6 \pm 6,30 a$ & - & - \\
\hline 6 & - & - & - & - & $40,8 \pm 7,00 a b$ & - & - \\
\hline 7 & - & - & - & - & $48,2 \pm 5,38 a b$ & - & - \\
\hline
\end{tabular}

Obs: As médias seguidas da mesma letra, não apresentam diferença significativa entre si, pelo teste de Tukey, ao nível de $5 \%$.

Tabela 5 - Longevidade média de machos de Aedes aegypti, em relação a diferentes períodos de quiescência.

\begin{tabular}{|c|c|c|c|c|c|c|c|}
\hline \multirow{2}{*}{$\begin{array}{l}\text { № do } \\
\text { grupo }\end{array}$} & \multicolumn{7}{|c|}{ Quiescência (dias) } \\
\hline & 3 & 32 & 63 & 91 & 121 & 154 & 427 \\
\hline 1 & $2,5 \pm 2,4$ & $24,7 \pm 1,8 a$ & $25,1 \pm 1,4 a$ & $35,8 \pm 2,3 a$ & $19,9 \pm 2,7 a$ & $24,5 \pm 0,0$ & $25,1 \pm 3,5$ \\
\hline 2 & - & $30,2 \pm 1,2 a$ & $23,6 \pm 1,5 a$ & - & $27,6 \pm 3,95 b$ & - & - \\
\hline 3 & - & $24,5 \pm 9,7 a$ & - & - & $57,8 \pm 4,85 a$ & - & - \\
\hline 4 & - & $39,1 \pm 2,37 a$ & - & - & $21,9 \pm 1,96 a$ & - & - \\
\hline 5 & - & - & - & - & $21,3 \pm 2,47 a$ & - & - \\
\hline 6 & - & - & - & - & $25,4 \pm 4,96 a b$ & - & - \\
\hline 7 & - & - & - & - & $16,1 \pm 1,44 a b$ & - & - \\
\hline
\end{tabular}

Obs: As médias seguidas da mesma letra, não apresentam diferença significativa entre si, pelo teste de Tukey, ao nível de $5 \%$. 


\section{DISCUSSÃO}

Existem na literatura várias teorias sobre o período de interrupção do desenvolvimento dos insetos, período que recebeu as denominações - diapausa e quiescência1 3492829 32. Com base nesses conceitos, optou-se pelo uso do termo quiescência, por mostrar-se esse mais adequado à experimentação feita a partir da secagem das cartelas de ovos recém ovipostos, por decréscimo de umidade.

A quiescência (interrupção no desenvolvimento induzida pela baixa umidade) é uma adaptação muito importante na dispersão passiva do $A$. aegypti, pois possibilita o transporte de ovos resistentes (ou quiescentes) em artefatos de todo tipo. A não destruição de pneus usados pode sinalizar um mecanismo factível do aumento da dispersão do $A$. aegypti, tornando imprescindível a atenção da vigilância entomológica ou epidemiológica. Esse contexto pode ganhar outra dimensão quando se adiciona o efeito altamente significativo da quiescência sobre a eclosão das larvas. Foram observados, neste trabalho com ovos quiescentes, períodos de até 720 dias, porém a viabilidade foi demonstrada até o período de 492 dias. Contudo o período de 121 dias apresentou a maior taxa de eclosão, que foi de $97,2 \%$. Este comportamento também foi observado em outro trabalho21 com períodos de 180 dias. Outros autores 79 tinham observado esse fenômeno, porém criaram outras denominações para esses períodos prolongados, tais como: ovos resistentes, duráveis, inativos e residuais.

Seja qual for a denominação utilizada, o fenômeno da interrupção provocada por variáveis climáticas, como o decréscimo de umidade e de temperatura, dá maiores perspectivas de vida ao A. aegypti por viabilizar o recrudescimento da espécie. Com relação ao controle desse mosquito este comportamento é relevante, uma vez que os ovos aderidos às paredes de recipientes resistem à dessecação, e eclodem mais tarde quando sobe o nível da água. Além disso, a quiescência deve servir de alerta à vigilância entomológica, assim como para indicar procedimento de cautela na divulgação de resultados sobre o controle ou erradicação desse mosquito, dentro daqueles períodos já mencionados.

Ovos quiescentes de $A$. aegypti apresentaram grande variação no período de incubação, sendo neste trabalho de 1 a 85 dias, com eclosões em blocos ou grupos, definindo os ciclos evolutivos.
Destes ciclos emergiam populações com sexagem equilibrada para o acasalamento, na razão aproximada de 1 para 1 . Esse comportamento tinha sido observado21 em trabalho com ciclos idênticos, e houve diferença apenas no período de incubação, que variou de 1 a 53 dias. Esse pode ser um mecanismo que - A. aegypti utiliza para colocar na natureza de forma contínua, embora flutuante, populações de adultos em diferentes períodos, quando as condições forem favoráveis, ou para se manter quando as condições forem adversas. A partir de capturas diárias², durante 12 meses, evidenciou-se a presença contínua do $A$. aegypti em Goiânia, apresentando uma flutuação com maiores densidades desse mosquito nas estações de verão e outono. O fenômeno da eclosão em grupos dentro dos períodos de quiescência, pode ser, neste caso, a explicação aceitável para a presença contínua e flutuante do $A$. aegypti.

A partir das eclosões em grupos, a duração do período larval e pupal mostrou-se estatisticamente igual, apresentando variações nos estádios larvares, curiosamente com alternância na duração, pois, quando se prolongava num estádio, ocorria a diminuição no outro, de forma a compensar a perda e terminar o desenvolvimento sem diferença significativa. Assim, os ciclos diferiram entre si em 99,8\%, pela ação determinante da incubação. Em condições climáticas similares a deste trabalho observouse o mesmo comportamento trabalhando com ovos que tinham 121 dias de quiescência, e obtiveram-se 5 grupos ou ciclos basicamente idênticos 21 .

O desenvolvimento pós-embrionário do A. aegypti, nos ciclos estudados, variou de 7 a 10 dias. Este desenvolvimento foi muito similar aos dados encontrados por outros pesquisadores 92123 , que foram entre 6 e 11 dias.

Entre esses períodos de quiescência, o de 121 dias foi o mais favorável ao $A$. aegypti, apresentando taxa de eclosão e número de ciclos (grupos) significativamente maiores do que nos outros períodos.

No acompanhamento de todos os ciclos evolutivos observou-se que as larvas comiam as exúvias ficando apenas as cápsulas cefálicas. Este comportamento foi mais freqüente nas larvas de $3^{\circ}$ e 4ํe estádios, sendo neste último, a ingestão de aproximadamente $100 \%$. De acordo com alguns autores 42932 , o tegumento dos 
insetos, principalmente os aquáticos, tem em sua camada externa uma cutícula composta de quitina, como elemento predominante, esclerotina, resilina e óxido de cálcio. Esses autores mostraram que na fisiologia da muda ou ecdise há reabsorção de quase todo o tegumento antes da ecdise, e elimina-se apenas a epicutícula. Esta é perdida e renovada em cada fase do desenvolvimento (crescimento); desta forma, cada muda representa, ainda, uma perda de nitrogênio e óxido de cálcio e de outras substâncias contidas na exúvia. O hábito da ingestão de exúvias, principalmente em $L_{3}$ e $L_{4}$, talvez seja uma maneira rápida e mais fácil de reposição dessas substâncias, principalmente quando se trabalha com dietas artificiais, não específicas.

\section{REFERÊNCIAS BIBLIOGRÁFICAS}

1. Andrewartha HG, Birch LC. The distribuition and abundance of animals. University of Chicago Press, Chicago, 1954.

2. Camargo MF, Silva IG, Elias CN, Silva HHG, Irata $Y$, Lemos SPS, Antunes SM, Fernandes FF. Diversidade e flutuação da entomofauna de Diptera Nematocera, na cidade de Goiânia, GO. Revista de Patologia Tropical 23:321, 1994.

3. Carvalho MB, Arruda EC, Arruda GP. Glossário de Entomologia. 2ª edição. UFPR, 1977.

4. Chapman RF. Diapause. In: The insects Structure and Function. The English Universities Press, London, p.717$731,1969$.

5. Chippaux A, Deubel V, Moreau JP, Reynes JM. Atual situação da febre amarela na América Latina. Bulletin de la Société de Pathologie Exotique 86:460-464, 1993.

6. Chungue E, Laudon F, Giaziou P. Dengue and dengue haemorrhagic fever in French Polynesia. Current situation. Tropical Medicine 35:209-215, 1993.

7. Cristophers, SR. Aedes aegypti. The yellow fever mosquito. Its life history, bionomics and structure. Cambridge Universities Press, London, 1960.

8. Fernandes FF, Silva IG, Camargo MF, Elias CN, Ação da deltametrina na dosagem de $0,8 \mathrm{~g} / \mathrm{Ha}$ sobre o Aedes aegypti (Lin., 1762) na formulação de ultra-baixo-volume. Revista de Patologia Tropical 26:37-45, 1997.

9. Forattini OP. Entomologia Médica. Vol. 2, EDUSP, São Paulo, 1965.

10. Franco O. História da febre amarela no Brasil. DNERu, Rio de Janeiro, RJ, 1969

11. Halstead SB. Global epidemiology of dengue: health systems in disarray. Tropical Medicine 35:137-146, 1993.

12. Khiem HB, Ha DQ, Huong VTQ, Loan, HTK. Some recent data on dengue epidemic in the South of Vietnam. Tropical Medicine 35:185-187, 1993.

13. Khin MN, Jirakanjanakit N, Yoksan S, Bhamarapravati N. Infection, dissemination, transmission, and biological attributes of dengue-2 PDK53 candidate vaccine virus oral infection in Aedes aegypti. American Journal Tropical Medicine and Hygiene 51:864-869, 1994.

14. Kitamura T. A rapporteur's summary:research on dengue vaccine. Tropical Medicine 35:325-330, 1993.

15. Miagostovich MP, Schatzmayr HG, Nogueira RMR, Lanciotti RS. Epidemiologia molecular de amostras de vírus dengue do Brasil. Revista da Sociedade Brasileira de Medicina Tropical 29(supl I):54, 1996.

16. Moreira TMS, Almeida MCM. Índices de infestação predial de A. aegypti e A. albopictus em Minas Gerais, 1993. Revista da Sociedade Brasileira de Medicina Tropical 27(supl I):363, 1994.

17. Okabe N. Situation on dengue fever and dengue haemorrhagic fever in the Western Pacific Region. Tropical Medicine 35:147-160, 1993.

18. Organização Mundial de Saúde. Dengue hemorrágico: diagnóstico, tratamento e controle. Genebra, 1987.

19. Oya A. Summarization of the Symposium. Tropical Medicine 35:341-342, 1993.

20. Quijivix C, Escalante L, Mata F, Barrios LC, Ogata K, Tada I. Dengue fever in Guatemala. Tropical Medicine 35:229-230, 1993.

21. Silva HHG, Silva IG, Elias CN, Lemos SPS, Rocha AP. Idade fisiológica de Aedes (Stegomyia) aegypti (Linnaeus, 1762) (Diptera, Culicidae). Revista de Patologia Tropical 24:269-273, 1995.

22. Silva IG, Araújo ESO, Silva HHG, Soares AW, Cantuária PB. Ocorrência de Aedes (Stegomyia) aegypti (Linnaeus, 1762) (Diptera, Culicidae) em Goiânia. Anais da Sociedade Entomológica do Brasil 20:459-460, 1991.

23. Silva IG, Camargo MF, Elias M, Elias CN. Ciclo evolutivo de Aedes (Stegomyia) aegypti (Linnaeus, 1762) (Diptera, Culicidae). Revista de Patologia Tropical 22:43-48, 1993.

24. Silva IG, Camargo MF, Elias CN, Isac E, Santos AH. Metodologia de criação de Aedes (Stegomyia) aegypti (Linnaeus, 1762) (Diptera, Culicidae) em condições de laboratório. Revista Goiana de Medicina 39:23-26, 1994.

25. Silva IG, Camargo MF, Guimarães FL, Elias M, Oliveira AWS. Estudo da eficácia da deltametrina (K-Othrine UBV $0,4 \%$ e $1 \%$ ) no combate ao Aedes (Stegomyia) aegypti (Linnaeus, 1762) e ao Culex quinquefasciatus (Wiedmann, 1828) (Diptera, Culicidae). Revista de Patologia Tropical 22:49-56, 1993.

26. Silva IG, Camargo MF, Silva HHG, Guimarães FL, Elias M, Oliveira AWS. Estudo da eficácia do Cythion no combate ao Aedes (Stegomyia) aegypti (Linnaeus, 1762) (Diptera, Culicidae). Revista Goiana de Medicina 39:13-16, 1994. 
27. Silva IG, Cantuária PB, Silva HHG, Araújo ESO. Distribuição de Aedes (Stegomyia) aegypti (Linnaeus, 1762) (Diptera, Culicidae) em Goiânia. Revista Patologia Tropical 20:1-5, 1991.

28. Silveira-Neto S, Nakano O, Barbin O, Villa-Nova NA. Fatores ecológicos. In: Manual de Ecologia dos Insetos. Ed. Agronômica Ceres Ltda, p.34-76, 1976.

29. Snodgrass RE. The organs of distribution, conservation, and elimination. In: Principles of insect morphology. Mc Graw-Hill Book, New York, p.381-421, 1935.

30. Soedarmo SP. The epidemiology, control and prevention of dengue haemorrhagic fever (DHF) in Indonésia. Tropical Medicine 35: 161-172, 1993.
31. Sucharit S, Rongsriyam Y, Deesin V, Komalamisra N, Apiwathnasorn C, Surathint K. Biology of dengue vectors and their control in Thailand. Tropical Medicine 35:253257, 1993.

32. Wigglesworth VB. Growth. In: Wigglesworth VB (ed) Insect Physiology. Toppan, Tokyo, p.96-113, 1974.

33. Yasui K. Strategies of dengue vaccine development by W.H.O. Using new biotechnology. Tropical Medicine 35:233-241, 1993. 